\title{
Non Degeneration of Fibonacci Series, Pascal's Elements and Hex Series
}

\author{
Balasubramani Prema Rangasamy \\ Ramanujan Institute for Advanced Study in Mathematics, University of Madras, Chennai, India \\ Email: balguve@gmail.com
}

How to cite this paper: Rangasamy, B.P. (2020) Non Degeneration of Fibonacci Series, Pascal's Elements and Hex Series. $A d$ vances in Pure Mathematics, 10, 393-404. https://doi.org/10.4236/apm.2020.107024

Received: June 6, 2020

Accepted: July 18, 2020

Published: July 21, 2020

Copyright $\odot 2020$ by author(s) and Scientific Research Publishing Inc. This work is licensed under the Creative Commons Attribution International License (CC BY 4.0).

http://creativecommons.org/licenses/by/4.0/

\begin{abstract}
Generally Fibonacci series and Lucas series are the same, they converge to golden ratio. After I read Fibonacci series, I thought, is there or are there any series which converges to golden ratio. Because of that I explored the inter relations of Fibonacci series when I was intent on Fibonacci series in my difference parallelogram. In which, I found there is no degeneration on Fibonacci series. In my thought, Pascal triangle seemed like a lower triangular matrix, so I tried to find the inverse for that. In inverse form, there is no change against original form of Pascal elements matrix. One day I played with ring magnets, which forms hexagonal shapes. Number of rings which forms Hexagonal shape gives Hex series. In this paper, I give the general formula for generating various types of Fibonacci series and its non-degeneration, how Pascal elements maintain its identities and which shapes formed by hex numbers by difference and matrices.
\end{abstract}

\section{Keywords}

Fibonacci Series, Lucas Series, Golden Ratio, Various Type of Fibonacci Series Generated by Matrices, Matrix Operations on Pascal's Elements and Hex Numbers

\section{Introduction}

The Fibonacci sequence is named after Leonardo of Pisa (c. 1170-c. 1250), popularly known as Fibonacci. He wrote a number of books such as Liber Abaci (The Book of Calculating) in 1202, Practica Geometriae (Practical Geometry) in 1220, Flos in 1225, and Liber Quadratorum (The Book of Squares) in 1225. Fibonacci sequence is a series of numbers in which each number is the sum of the two preceding numbers. First few numbers in the series are 1, 1, 2, 3, 5, 8, 13, 21, 34,55 ... In India, Fibonacci sequence appeared in Sanskrit prosody (a system of 
versification). In the Sanskrit oral tradition, there was much emphasis on how long (L) syllables that are 2 units of duration mix with the short (S) syllables that are 1 unit of duration. Counting the different patterns of $\mathrm{L}$ and $\mathrm{S}$ within a given fixed length results in the Fibonacci numbers-the number of patterns that are $m$ short syllables-long is the Fibonacci number $F m+1$. According to Susantha Goonatilake of Royal Asiatic Society Sri Lanka, the development of the Fibonacci sequence "is attributed in part to Pingala (200 BC), later being associated with Virahanka (c. AD 700), Gopala (c. AD 1135), and Hemchandra (c.AD 1150)". To find $F n$ for a general positive integer $n$, we hope that we can see a pattern in the sequence of numbers already found. A sharp eye can now detect that any number in the sequence is always the sum of the two numbers preceding it. That is,

$$
F_{n+2}=F_{n+1}+F_{n}, \text { for } n=0,1,2,3, \cdots .
$$

Fibonacci series is helix like identity. It converges to golden ratio, we can show its existence in spiral shells but its elements never construct volumetric object. Fibonacci series elements construct Area only. Pascal triangle elements (Binomial series elements) construct area, volume and volumetric objects but whatever be it remains its identity which means, if we constructed a matrix with Pascal triangle elements, which would be a square matrix, its $k^{\text {th }}$ power or its inverse might have the same identity of Pascal triangle elements, hex series having different numbers, but all numbers will be derived by triangular series numbers.

The Fibonacci Numbers are also applied in Pascal's Triangle. Entry is sum of the two numbers either side of it, but in the row above. Diagonal sums in Pascal's Triangle are the Fibonacci numbers. We are getting some ideas from ([1] Jeffrey R. Chasnov (2016-19) - Fibonacci Numbers and the Golden ratio - Lecture Notes for Course - The Hong Kong University of Science and Technology, Department of Mathematics, Clear Water Bay, Kowloon - Hong Kong). We know Fibonacci Numbers and the Golden ratio ([2] Tom Davis, Exploring Pascals Triangle- tomrdavis@earthlink.net http://www.geometer.org/mathcircles, January 1, 2010; Relation between Pascal's triangle and Fibonacci's numbers; [3] Balasubramani Prema Rangasamy - Some extensions on numbers - Advances in Pure Mathematics, 2019, 9, 944-958. Difference table and [4] https://en.wikipedia.org/w/index.php?title=Golden ratio\&oldid=83746951"8) We know more about Fibonacci's elements, Pascal's elements, Hex numbers and Golden ratio. The Golden Section represented by the Greek letter Phi $(\varphi)=$ 1.6180339887.

In this paper, I give the general formula for generating various types of Fibonacci series and its non-degeneration, how Pascal elements maintain its identities and which shapes formed by hex numbers by difference and matrices.

\section{Row Matrix Building for Fibonacci's Elements}

\section{Difference method}




$$
\left[\begin{array}{cc}
1 & 1 \\
1 & 2 \\
2 & 3 \\
3 & 5 \\
5 & 8 \\
8 & 13 \\
13 & 21 \\
\vdots & \vdots
\end{array}\right] \text { is a } m \times 2 \text { matrix. }
$$

In which odd rows numbers are Fibonacci series numbers and even rows numbers are difference of two consecutive odd rows numbers.

$$
\left[\begin{array}{ccc}
1 & 1 & 2 \\
2 & 4 & 6 \\
3 & 5 & 8 \\
10 & 16 & 26 \\
13 & 21 & 34 \\
42 & 68 & 110 \\
55 & 89 & 144 \\
\vdots & \vdots & \vdots
\end{array}\right] \text { is a } m \times 3 \text { matrix. }
$$

In which odd rows numbers are Fibonacci series numbers and even rows numbers are difference of two consecutive odd rows numbers.

$$
\left[\begin{array}{cccc}
1 & 1 & 2 & 3 \\
4 & 7 & 11 & 18 \\
5 & 8 & 13 & 21 \\
29 & 47 & 76 & 123 \\
34 & 55 & 89 & 144 \\
199 & 322 & 521 & 843 \\
233 & 377 & 610 & 987 \\
\vdots & \vdots & \vdots & \vdots
\end{array}\right] \text { is a } m \times 4 \text { matrix. }
$$

In which odd rows numbers are Fibonacci series numbers and even rows numbers are difference of two consecutive odd rows numbers.

$$
\left[\begin{array}{ccccc}
1 & 1 & 2 & 3 & 5 \\
7 & 12 & 19 & 31 & 50 \\
8 & 13 & 21 & 34 & 55 \\
81 & 131 & 212 & 343 & 555 \\
89 & 144 & 233 & 377 & 610 \\
898 & 1453 & 2351 & 3804 & 6155 \\
987 & 1597 & 2584 & 4181 & 6765 \\
\vdots & \vdots & \vdots & \vdots & \vdots
\end{array}\right] \text { is a } m \times 5 \text { matrix. }
$$

In which odd rows numbers are Fibonacci series numbers and even rows numbers are difference of two consecutive odd rows numbers. 


\begin{tabular}{llllllllllll}
\multicolumn{10}{l}{ We do the same again and again we get } \\
$\begin{array}{lllllllllllll}\text { st } \\
1\end{array}$ series: & 1 & 1 & 2 & 3 & 5 & 8 & 13 & 21 & 34 & 55 & 89 \\
$2^{\text {nd }}$ series: & 1 & 2 & 3 & 5 & 8 & 13 & 21 & 34 & 55 & 89 & 144 \\
$3^{\text {rd }}$ series: & 2 & 4 & 6 & 10 & 16 & 26 & 42 & 68 & 110 & 178 & 288 \\
$4^{\text {th }}$ series: & 4 & 7 & 11 & 18 & 29 & 47 & 76 & 123 & 199 & 322 & 521 \\
$5^{\text {th }}$ series: & 7 & 12 & 19 & 31 & 50 & 81 & 131 & 212 & 343 & 555 & 898 \\
$6^{\text {th }}$ series: & 12 & 20 & 32 & 52 & 84 & 136 & 220 & 356 & 576 & 932 & 1508 \\
\hline
\end{tabular}

We can generate $m^{\text {th }}$ series by

$$
{ }^{m} F_{n+2}={ }^{m} F_{n+1}+{ }^{m} F_{n}
$$

where

$$
{ }^{m} F_{n}={ }^{1} F_{m+n}-{ }^{1} F_{n} \text { and } m \text { is an } m^{\text {th }} \text { Fibonacci's series. }
$$

where ${ }^{m} F_{n}$ an $n^{\text {th }}$ element of a $m^{\text {th }}$ Fibonacci series, ${ }^{1} F_{n}$ is $n^{\text {th }}$ element of a $1^{\text {st }}$ Fibonacci series and ${ }^{1} F_{m+n}$ is $m+n^{\text {th }}$ element of a $1^{\text {st }}$ Fibonacci series.

$1^{\text {st }}$ series elements are known as Fibonacci numbers.

$4^{\text {th }}$ series elements are known as Lucas numbers.

Axiom 1: All the above series are converges to Golden ratio.

\section{Addition Method}

$$
\left[\begin{array}{cc}
1 & 1 \\
3 & 4 \\
2 & 3 \\
7 & 11 \\
5 & 8 \\
18 & 29 \\
13 & 21 \\
\vdots & \vdots
\end{array}\right] \text { is a } m \times 2 \text { matrix. }
$$

In which odd rows numbers are Fibonacci series numbers and even rows numbers are addition of two consecutive odd rows numbers.

$$
\left[\begin{array}{ccc}
1 & 1 & 2 \\
4 & 6 & 10 \\
3 & 5 & 8 \\
16 & 26 & 42 \\
13 & 21 & 34 \\
68 & 110 & 178 \\
55 & 89 & 144 \\
\vdots & \vdots & \vdots
\end{array}\right] \text { is a } m \times 3 \text { matrix. }
$$

In which odd rows numbers are Fibonacci series numbers and even rows numbers are addition of two consecutive odd rows numbers. 


$$
\left[\begin{array}{cccc}
1 & 1 & 2 & 3 \\
6 & 9 & 15 & 24 \\
5 & 8 & 13 & 21 \\
39 & 63 & 102 & 165 \\
34 & 55 & 89 & 144 \\
267 & 432 & 699 & 1131 \\
233 & 377 & 610 & 987 \\
\vdots & \vdots & \vdots & \vdots
\end{array}\right] \text { is a } m \times 4 \text { matrix. }
$$

In which odd rows numbers are Fibonacci series numbers and even rows numbers are addition of two consecutive odd rows numbers.

$$
\left[\begin{array}{ccccc}
1 & 1 & 2 & 3 & 5 \\
9 & 14 & 23 & 37 & 60 \\
8 & 13 & 21 & 34 & 55 \\
97 & 157 & 254 & 411 & 665 \\
89 & 144 & 233 & 377 & 610 \\
1076 & 1741 & 2817 & 4558 & 7375 \\
987 & 1597 & 2584 & 4181 & 6765 \\
\vdots & \vdots & \vdots & \vdots & \vdots
\end{array}\right] \text { is a } m \times 5 \text { matrix. }
$$

In which odd rows numbers are Fibonacci series numbers and even rows num-

\begin{tabular}{|c|c|c|c|c|c|c|c|c|c|c|c|}
\hline \multicolumn{12}{|c|}{ We do the same again and again we get } \\
\hline $1^{\text {st }}$ series: & 1 & 1 & 2 & 3 & 5 & 8 & 13 & 21 & 34 & 55 & 89 \\
\hline $2^{\text {nd }}$ series: & 3 & 4 & 7 & 11 & 18 & 29 & 47 & 76 & 123 & 199 & 322 \\
\hline $3^{\text {rd }}$ series: & 4 & 6 & 10 & 16 & 26 & 42 & 68 & 110 & 178 & 288 & 466 \\
\hline $4^{\text {th }}$ series: & 6 & 9 & 15 & 24 & 39 & 63 & 102 & 165 & 267 & 432 & 699 \\
\hline $5^{\text {th }}$ series: & 9 & 14 & 23 & 37 & 60 & 97 & 157 & 254 & 411 & 665 & 1076 \\
\hline $6^{\text {th }}$ series: & 14 & 22 & 36 & 58 & 94 & 152 & 246 & 398 & 644 & 1042 & 168 \\
\hline
\end{tabular}
bers are addition of two consecutive odd rows numbers.

We can generate $k^{\text {th }}$ series by

$$
{ }^{k} F_{k+2}={ }^{k} F_{n+1}+{ }^{k} F_{n}
$$

and

$$
{ }^{k} F_{n}={ }^{1} F_{k+n}+{ }^{1} F_{n}
$$

where ${ }^{k} F_{n}$ an $n^{\text {th }}$ element of a $k^{\text {th }}$ Fibonacci series, ${ }^{1} F_{n}$ is $n^{\text {th }}$ element of a $1^{\text {st }}$ Fibonacci series and ${ }^{1} F_{k+n}$ is $k+1^{\text {th }}$ element of a $1^{\text {st }}$ Fibonacci series.

$1^{\text {st }}$ series elements are known as Fibonacci numbers.

$2^{\text {nd }}$ series elements are known as Lucas numbers.

$3^{\text {rd }}$ series elements are known as doubled Fibonacci numbers.

$4^{\text {th }}$ series elements are known as tripled Fibonacci numbers. 


\section{Difference between All Series Diagonal Elements}

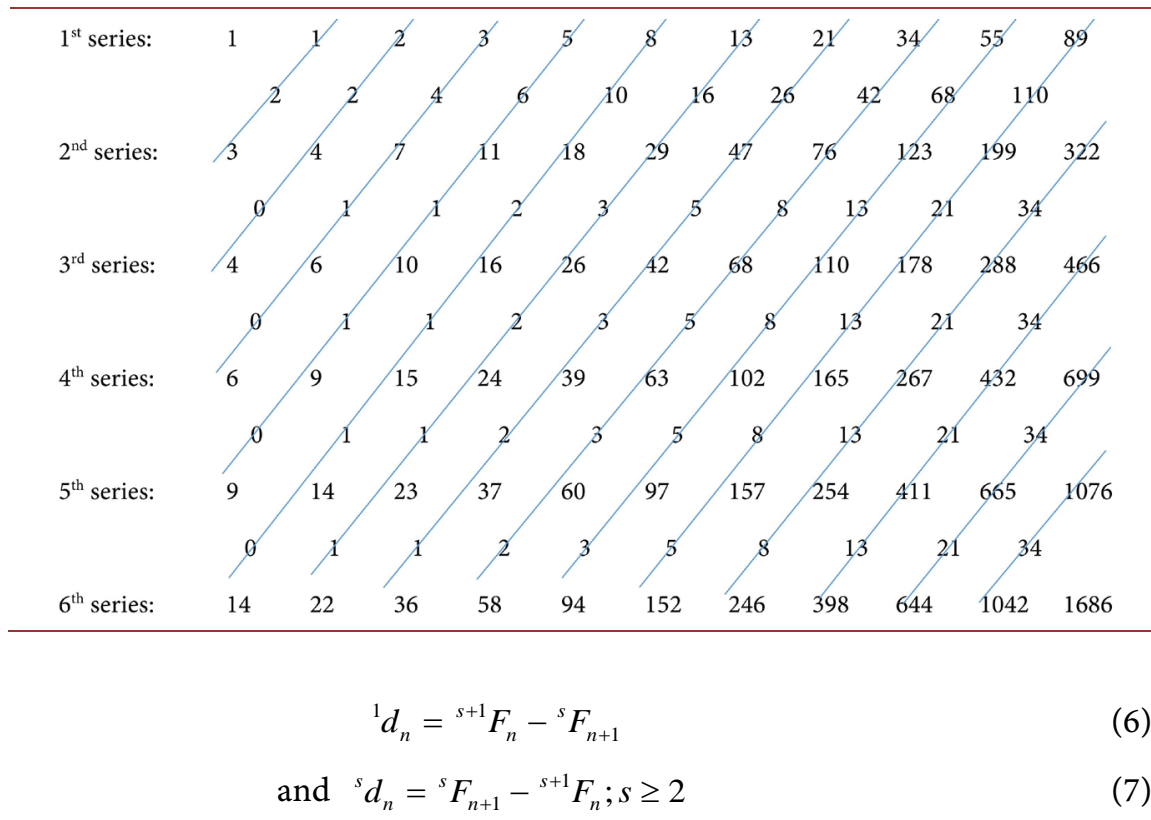

where ${ }^{s} d_{n}$ is $n^{\text {th }}$ element of a $s^{\text {th }}$ different series, ${ }^{s+1} F_{n}$ is $n^{\text {th }}$ element of a $s+1^{\text {th }}$ Fibonacci series and ${ }^{s} F_{n+1}$ is $n+1^{\text {th }}$ element of a $s^{\text {th }}$ Fibonacci series.

From above those diagonal differences remains the extinct of Fibonacci's elements.

\section{Difference Chart of Above Series}

\begin{tabular}{|c|c|c|c|c|c|c|c|}
\hline & & Diff. 1 & Diff. 2 & Diff. 3 & Diff. 4 & $\ldots$ & \\
\hline \multirow[t]{2}{*}{$1^{\text {st }}$ series: } & 1 & 1 & 2 & 3 & 5 & & 8 \\
\hline & & 0 & 1 & 1 & 2 & 3 & \\
\hline \multirow[t]{2}{*}{$2^{\text {nd }}$ series: } & 1 & 2 & 3 & 5 & 8 & & 13 \\
\hline & & 1 & 1 & 2 & 3 & 5 & \\
\hline \multirow[t]{2}{*}{$3^{\text {rd }}$ series: } & 2 & 4 & 6 & 10 & 16 & & 26 \\
\hline & & 2 & 2 & 4 & 6 & 10 & \\
\hline \multirow[t]{2}{*}{$4^{\text {th }}$ series: } & 4 & 7 & 11 & 18 & 29 & & 47 \\
\hline & & 3 & 4 & 7 & 11 & 18 & \\
\hline \multirow[t]{2}{*}{$5^{\text {th }}$ series: } & 7 & 12 & 19 & 31 & 50 & & 81 \\
\hline & & 5 & 7 & 12 & 19 & 31 & \\
\hline \multirow[t]{2}{*}{$6^{\text {th }}$ series: } & 12 & 20 & 32 & 52 & 84 & & 136 \\
\hline & & 8 & 12 & 20 & 32 & 52 & \\
\hline \multirow[t]{2}{*}{$7^{\text {th }}$ series: } & 20 & 33 & 53 & 86 & 139 & & 225 \\
\hline & & 13 & 20 & 33 & 53 & 86 & \\
\hline
\end{tabular}

From the above we chart,

\begin{tabular}{lllllll}
\hline & Series 1 & series 2 & series 3 & series 4 & series 5 & $\ldots$ \\
\hline Diff 1 series: & 0 & 1 & 2 & 3 & 5 & $\ldots$ \\
Diff 2 series: & 1 & 1 & 2 & 4 & 7 & $\ldots$ \\
Diff 3 series: & 1 & 2 & 4 & 7 & 12 & $\ldots$ \\
Diff 4 series: & 2 & 3 & 6 & 11 & 19 & $\ldots$ \\
Diff 5 series: & 3 & 5 & 10 & 18 & 31 & $\ldots$ \\
\hline
\end{tabular}


Diff $t^{\text {th }}$ series:

$$
{ }^{t+2} D_{n}={ }^{t+1} D_{n}+{ }^{t} D_{n}
$$

where

$$
\begin{aligned}
{ }^{t} D_{n} & ={ }^{t} F_{n+1}-{ }^{t} F_{n} \\
\text { and }{ }^{t+1} D_{n} & ={ }^{t+1} F_{n+1}-{ }^{t+1} F_{n}
\end{aligned}
$$

where ${ }^{t} D_{n}$ an $n^{\text {th }}$ element of a $t^{\text {th }}$ different Fibonacci series, ${ }^{1} F_{n}$ is $n^{\text {th }}$ element of a $1^{\text {st }}$ Fibonacci series and ${ }^{1} F_{k+n}$ is $k+1^{\text {th }}$ element of a $1^{\text {st }}$ Fibonacci series.

\begin{tabular}{|c|c|c|c|c|c|c|c|c|c|c|c|c|c|c|c|c|c|c|c|c|c|c|c|c|c|}
\hline \multirow{8}{*}{$\begin{array}{lll}0 & \\
& \\
& 1\end{array}$} & 1 & & 1 & & 2 & & 3 & & 5 & & 8 & & 13 & & 21 & & 34 & & $\ldots$ & & & & & & \\
\hline & & 0 & & 1 & & 1 & & 2 & & 3 & & 5 & & 8 & & 13 & & 21 & & $\ldots$ & & & & & \\
\hline & -1 & & 1 & & 0 & & 1 & & 1 & & 2 & & 3 & & 5 & & 8 & & 13 & & $\ldots$ & & & & \\
\hline & & 2 & & -1 & & 1 & & 0 & & 1 & & 1 & & 2 & & 3 & & 5 & & 8 & & $\ldots$ & & & \\
\hline & & & -3 & & 2 & & -1 & & 1 & & 0 & & 1 & & 1 & & 2 & & 3 & & 5 & & $\ldots$ & & \\
\hline & & & & 5 & & -3 & & 2 & & -1 & & 1 & & 0 & & 1 & & 1 & & 2 & & 3 & & $\ldots$ & \\
\hline & & & & & -8 & & 5 & & -3 & & 2 & & -1 & & 1 & & 0 & & 1 & & 1 & & 2 & & .. \\
\hline & & & & & & 13 & & -8 & & 5 & & -3 & & 2 & & -1 & & 1 & & 0 & & 1 & & 1 & \\
\hline & & & & & & & $\ldots$ & & $\ldots$ & & $\ldots$ & & $\ldots$ & & $\ldots$ & & $\ldots$ & & $\ldots$ & & $\ldots$ & & $\ldots$ & &.. \\
\hline
\end{tabular}

Axiom 3: All the above different series are converges to Golden ratio.

\section{Difference Parallelogram of Fibonacci Numbers}

Above difference parallelogram shows Fibonacci series never vanished, which means it exist everlastingly.

\section{Matrices in Pascal's Elements}

Let

$$
A=\left[\begin{array}{cccccc}
1 & & & & & \\
1 & 1 & & & & \\
1 & 2 & 1 & & & \\
1 & 3 & 3 & 1 & & \\
\vdots & \vdots & \vdots & \vdots & \ddots & \\
{ }^{m} C_{0} & { }^{m} C_{1} & { }^{m} C_{2} & { }^{m} C_{3} & \ldots & { }^{m} C_{m}
\end{array}\right]
$$

be an $n \times n$ matrix having Pascal's elements. Where $m=n-1$. We called it as Pascal's matrix.

Now we define Pascal matrix by any variable.

1) NW (North-west Pascal's matrix)

Let

$$
A=\left[\begin{array}{cccccc}
a & & & & & \\
a & a & & & & \\
a & 2 a & a & & & \\
a & 3 a & 3 a & a & & \\
\vdots & \vdots & \vdots & \vdots & \ddots & \\
{ }^{m} C_{0} a & { }^{m} C_{1} a & { }^{m} C_{2} a & { }^{m} C_{3} a & \ldots & { }^{m} C_{m} a
\end{array}\right]
$$


be an $n \times n$ matrix having Pascal's elements. Where $m=n-1 . k$ is an exponent and " $a$ " is variant.

Now,

$$
\begin{aligned}
& A^{k}=\left[\begin{array}{cccccc}
k^{0} a^{k} & & & & & \\
k^{1} a^{k} & k^{0} a^{k} & & & & \\
k^{2} a^{k} & 2 k^{1} a^{k} & k^{0} a^{k} & & & \\
k^{3} a^{k} & 3 k^{2} a^{k} & 3 k^{1} a^{k} & k^{0} a^{k} & & \\
\vdots & \vdots & \vdots & \vdots & \ddots & \\
{ }^{m} C_{0} k^{n-1} a^{k} & { }^{m} C_{1} k^{n-2} a^{k} & { }^{m} C_{2} k^{n-3} a^{k} & { }^{m} C_{3} k^{n-4} a^{k} & \ldots & { }^{m} C_{m} k^{0} a^{k}
\end{array}\right]
\end{aligned}
$$

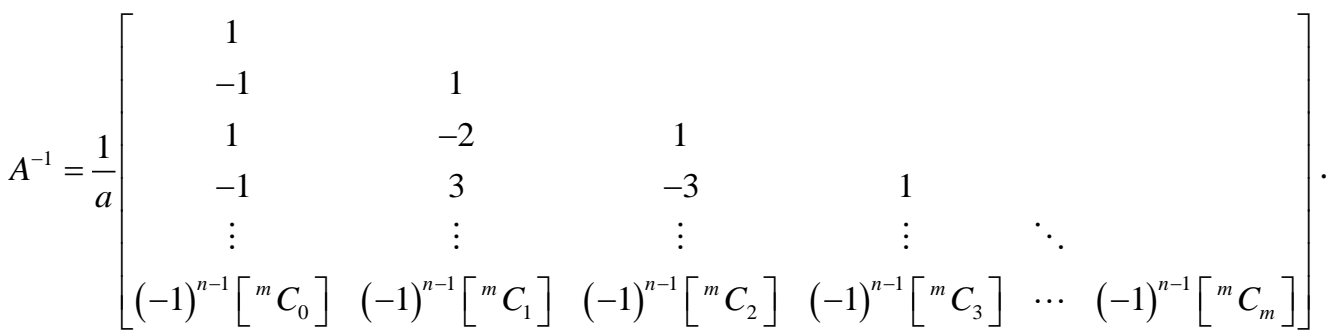

Jordan normal matrix of $A$

$$
J_{A}=\left[\begin{array}{cccccc}
a & 1 & 0 & 0 & 0 & 0 \\
0 & a & 1 & 0 & 0 & 0 \\
0 & 0 & a & 1 & 0 & 0 \\
0 & 0 & 0 & a & 1 & 0 \\
\vdots & \vdots & \vdots & \vdots & \ddots & 1 \\
0 & 0 & 0 & 0 & \cdots & a
\end{array}\right]
$$

\section{2) NE (North-East Pascal's matrix)}

Let

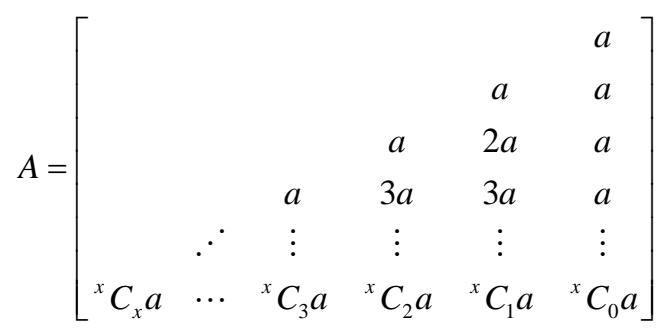

be an $y \times y$ matrix having Pascal's elements. Where $x=y-1 . k$ is an exponent and " $a$ " is variant.

Now, inverse for North-East matrix

$$
A^{-1}=\frac{1}{a}\left[\begin{array}{cccccc}
(-1)^{y-1}\left[{ }^{x} C_{0}\right] & (-1)^{y-1}\left[{ }^{x} C_{1}\right] & (-1)^{y-1}\left[{ }^{x} C_{2}\right] & (-1)^{y-1}\left[{ }^{x} C_{3}\right] & \cdots & (-1)^{y-1}\left[{ }^{x} C_{x}\right] \\
\vdots & \vdots & \vdots & \vdots & . & \\
-1 & 3 & -3 & 1 & & \\
1 & -2 & 1 & & & \\
-1 & 1 & & & &
\end{array}\right]
$$

3) SE (South-East Pascal's matrix) 
Let

$$
A=\left[\begin{array}{cccccc}
{ }^{m} C_{m} a & \ldots & { }^{m} C_{3} a & { }^{m} C_{2} a & { }^{m} C_{1} a & { }^{m} C_{0} a \\
& \ddots & \vdots & \vdots & \vdots & \vdots \\
& & a & 3 a & 3 a & a \\
& & & a & 2 a & a \\
& & & & a & a \\
& & & & & a
\end{array}\right]
$$

be an $n \times n$ matrix having Pascal's elements. Where $m=n-1 . k$ is an exponent and " $a$ " is variant.

Now,

$$
A^{-1}=\frac{1}{a}\left[\begin{array}{cccccc}
(-1)^{n-1}\left[{ }^{m} C_{m}\right] & & (-1)^{n-1}\left[{ }^{m} C_{3}\right] & (-1)^{n-1}\left[{ }^{m} C_{2}\right] & (-1)^{n-1}\left[{ }^{m} C_{1}\right] & (-1)^{n-1}\left[{ }^{m} C_{0}\right] \\
& \ddots & \vdots & \vdots & \vdots & \vdots \\
& & 1 & -3 & 3 & -1 \\
& & & 1 & -2 & 1 \\
& & & & 1 & -1
\end{array}\right] .
$$

4) SW (South-West Pascal's matrix)

Let

$$
A=\left[\begin{array}{cccccc}
{ }^{x} C_{0} a & { }^{x} C_{1} a & { }^{x} C_{2} a & { }^{x} C_{3} a & \ldots & { }^{x} C_{x} a \\
\vdots & \vdots & \vdots & \vdots & . & \\
a & 3 a & 3 a & a & & \\
a & 2 a & a & & & \\
a & a & & & & \\
a & & & & &
\end{array}\right]
$$

be an $y \times y$ matrix having Pascal's elements. Where $x=y-1 . k$ is an exponent and ' $a$ ' is variant.

Now, inverse for south-west matrix

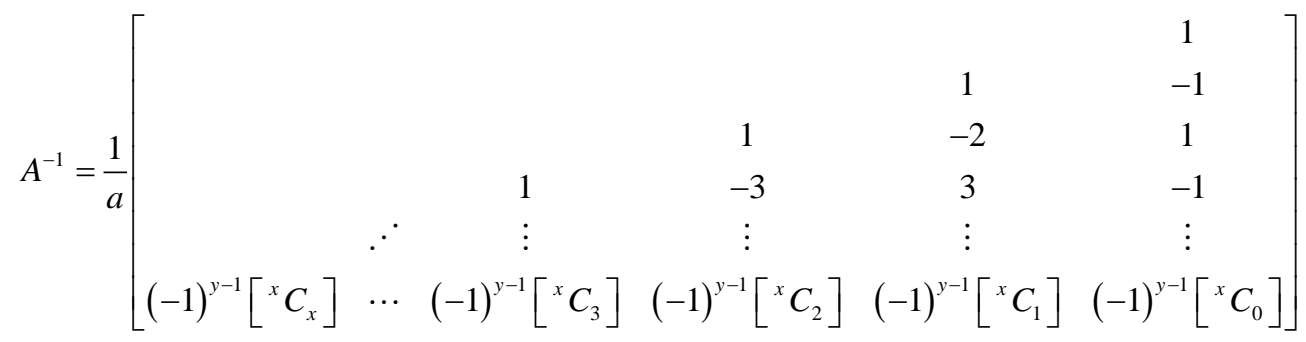

Hex numbers:

\begin{tabular}{ccccccccccc}
\hline 1 & 7 & 19 & 37 & 61 & 91 & 127 & 169 & 217 & 271 & 331 \\
397 & 469 & 547 & 631 & 721 & 817 & 919 & 1027 & 1141 & 1261 & $\ldots$ \\
\hline
\end{tabular}

Let $h$ be any hex number. We know mod 6 of any $h$ is equal to 1 .

Mod 6 of $h_{1} \equiv 1$; Mod 6 of $h_{2} \equiv 1 ; \cdots ; \operatorname{Mod} 6$ of $h_{k} \equiv 1$; 
Theorem 1: Difference between any two elements of Hex numbers is fully divided by 6 .

Theorem 2: $\sum_{k=0}^{\infty} H_{6 n \pm k} \equiv a \bmod 6$, where $n$ is integer and $0 \leq a<6$.

Theorem 3: Remainder of arbitrary product of any number of Hex series is always 1 when the product is divided by 6 .

Proof:

$$
R=\frac{\prod_{i} H_{i}}{6}=1
$$

We can say above as

$$
R\left(h_{1} \times h_{2} \times \cdots \times h_{k}\right) \div 6 \equiv(1 \times 1 \times \cdots \times 1) \bmod 6=1 .
$$

Theorem 4: $\sum_{o} H_{k} \bmod (6) \equiv \sum_{E} H_{k}(\bmod 6) \mid k \in Z$

Theorem 5: $\sum_{k=0}^{\infty} H_{k}=k^{3}$, where $H_{k}$ is Hex series elements.

Matrices of Hex numbers

Let we see the relation between hex numbers in matrix

1) Let $A=\left[\begin{array}{cc}1 & h+1 \\ 3 h+1 & 6 h+1\end{array}\right]$ be a $2 \times 2$ matrix which elements are hex numbers (where $h=6$ ) then $|A|=\left|\begin{array}{cc}1 & h+1 \\ 3 h+1 & 6 h+1\end{array}\right|=6 h+1-3 h^{2}-4 h+1=-3 h^{2}+2 h$

2) Let $A=\left[\begin{array}{cc}h+1 & 3 h+1 \\ 6 h+1 & 10 h+1\end{array}\right]$ be a $2 \times 2$ matrix which elements are hex numbers (where $h=6)$ then $|A|=\left|\begin{array}{cc}h+1 & 3 h+1 \\ 6 h+1 & 10 h+1\end{array}\right|=-8 h^{2}+2 h$

By above way we get, $-15 h^{2}+2 h ;-24 h^{2}+2 h ; \cdots ;-n(n+2) h^{2}+2 h$

\begin{tabular}{|c|c|c|c|c|c|c|c|}
\hline Initial: $-3 h^{2}+2 h$ & & $-8 h^{2}+2 h$ & $-15 h^{2}+2 h$ & & $-24 h^{2}+2 h$ & & $-35 h^{2}+2 h$ \\
\hline $1^{\text {st }}$ diff: & $5 h^{2}$ & $7 h^{2}$ & & $9 h^{2}$ & & $11 h^{2}$ & \\
\hline $2^{\text {nd }}$ diff: & & $2 h^{2}$ & $2 h^{2}$ & & $2 h^{2}$ & & $\ldots$ \\
\hline $3^{\text {rd }}$ diff: & & 0 & & 0 & & $\ldots$ & \\
\hline
\end{tabular}

1) Let we construct a difference triangle about above determinants

a) Let $A=\left[\begin{array}{ccc}1 & h+1 & 3 h+1 \\ 6 h+1 & 10 h+1 & 15 h+1 \\ 21 h+1 & 28 h+1 & 36 h+1\end{array}\right]$ be a $3 \times 3$ matrix which elements are hex numbers then $|A|=\left|\begin{array}{ccc}1 & h+1 & 3 h+1 \\ 6 h+1 & 10 h+1 & 15 h+1 \\ 21 h+1 & 28 h+1 & 36 h+1\end{array}\right|=-27 h^{3}$

b) $|A|=\left|\begin{array}{ccc}h+1 & 3 h+1 & 6 h+1 \\ 10 h+1 & 15 h+1 & 21 h+1 \\ 28 h+1 & 36 h+1 & 45 h+1\end{array}\right|=-27 h^{3}$ 
2) Let we construct a difference triangle about above determinants

\begin{tabular}{|c|c|c|c|c|c|c|c|c|c|c|}
\hline Initial: & $27 h^{3}$ & & $27 h^{3}$ & & $27 h^{3}$ & & $27 h^{3}$ & & $27 h^{3}$ & $\ldots$ \\
\hline $1^{\text {st }}$ diff: & & 0 & & 0 & & 0 & & 0 & & $\ldots$ \\
\hline
\end{tabular}

a) Let $A=\left[\begin{array}{cccc}1 & h+1 & 3 h+1 & 6 h+1 \\ 10 h+1 & 15 h+1 & 21 h+1 & 28 h+1 \\ 36 h+1 & 45 h+1 & 55 h+1 & 66 h+1 \\ 78 h+1 & 91 h+1 & 105 h+1 & 120 h+1\end{array}\right]$ be a $4 \times 4$ matrix which elements are hex numbers then $|A|=\left|\begin{array}{cccc}1 & h+1 & 3 h+1 & 6 h+1 \\ 10 h+1 & 15 h+1 & 21 h+1 & 28 h+1 \\ 36 h+1 & 45 h+1 & 55 h+1 & 66 h+1 \\ 78 h+1 & 91 h+1 & 105 h+1 & 120 h+1\end{array}\right|=0$

b) $|A|=\left|\begin{array}{cccc}h+1 & 3 h+1 & 6 h+1 & 10 h+1 \\ 15 h+1 & 21 h+1 & 28 h+1 & 36 h+1 \\ 45 h+1 & 55 h+1 & 66 h+1 & 78 h+1 \\ 91 h+1 & 105 h+1 & 120 h+1 & 136 h+1\end{array}\right|=0$

3) Let we construct a difference triangle about above determinants

\begin{tabular}{ccccccc}
\hline Initial: & 0 & 0 & 0 & 0 & 0 & $\ldots$ \\
\hline
\end{tabular}

From the above we can state:

a) Determinants of $2 \times 2$ matrix with hex series elements vanished at $2^{\text {nd }}$ difference.

b) Determinant of $3 \times 3$ matrix with hex series elements vanished at $0^{\text {th }}$ difference.

c) Determinant of $4 \times 4$ matrix with hex series elements and above are 0 .

Which means hex series elements are forming hexagonal only.

\section{Conclusions}

1) Fibonacci series never dies. We can generate so many series like Fibonacci series, they also converge to golden ratio. By this way we find so many golden ratio pairs.

2) Matrix with Pascal elements never vanished at any " $n$ " dimensional matrix calculation. For all arithmetic and matrix operation of matrix with Pascal elements never give up its frame. Here frame means the structure of matrix.

3) Sum of $k^{\text {th }}$ elements of hex series gives $k^{3}$ and hex series elements form hexagonal only.

\section{Conflicts of Interest}

The author declares no conflicts of interest regarding the publication of this paper.

\section{References}

[1] Chasnov, J.R. (2016) Fibonacci Numbers and the Golden Ratio. Lecture Notes for 
Course, The Hong Kong University of Science and Technology, Department of Mathematics, Clear Water Bay, Kowloon, Hong Kong.

[2] Davis, T. (2010) Exploring Pascal's Triangle.

http://www.geometer.org/mathcircles

[3] Rangasamy, B.P. (2019) Some Extensions on Numbers. Advances in Pure Mathematics, 9, 944-958. https://doi.org/10.4236/apm.2019.911047

[4] https://en.wikipedia.org/w/index.php?title=Golden ratio\&oldid=83746951"8 